\title{
Applications of Optical Imaging System in Dentistry
}

Joo Beom Eom ${ }^{1}$

Anjin Park ${ }^{2}$

${ }^{1}$ Department of Biomedical Science, College of Medicine, Dankook University, Cheonan, Korea ${ }^{2}$ Medical Photonics Research Center, Korea Photonics Technology Institute, Gwangju, Korea

\section{Correspondence}

Joo Beom Eom

Department of Biomedical Science, College of Medicine, Dankook University, 119 Dandae-ro, Dongnam-gu, Cheonan 31116, Korea

Tel.: +82-41-550-3275

Fax: +82-41-550-3275

E-mail: jbeomadankook.ac.kr

(C) Korean Society for Laser Medicine and Surgery

(c) This is an open access article distributed under the terms of the Creative Commons Attribution NonCommercial License (http://creativecommons.org/ licenses/by-nc/4.0) which permits unrestricted noncommercial use, distribution, and reproduction in any medium, provided the original work is properly cited.

\begin{abstract}
Optical-based imaging technology has high resolution and can assess images in real time. Numerous studies have been conducted for its application in the dental field. The current research introduces an oral camera that includes fluorescent imaging, a second study examining a 3D intraoral scanner applying a confocal method and a polarization structure that identifies the 3D image of a tooth, and finally, an optical coherence tomography technique. Using this technique, we introduce a new concept 3D oral scanner that simultaneously implements 3D structural imaging as well as images that diagnose the inside of teeth. With the development of light source technology and detector technology, various optical-based imaging technologies are expected to be applied in dentistry.
\end{abstract}

\section{Key words}

Optical imaging; Intraoral camera; Intraoral scanner; Optical coherence tomography; Dentistry 


\section{INTRODUCTION}

Currently, many studies have been conducted to apply the technique of diagnosis to a dentist using an optical method that has high resolution and can acquire images in real time. The representative diagnostic device of the optical method is an oral camera. It uses a white light source (representatively LED) and is equipped with a compact camera module to implement images in the oral cavity as color images, and communicate with patients using diagnostic images. The oral camera has been developed as an oral camera for dental caries diagnosis using the green fluorescence feature of teeth by adding a fluorescent imaging technique..$^{1-7}$ In order to acquire a 3D image of a tooth and apply it to a prosthesis, research on a 3D intraoral scanner has been active and commercialized. Many research groups are conducting research not only for the diagnosis of the surface of the teeth, but also for the diagnosis of the inside of the teeth. In order to check the lesions inside the teeth, studies have been conducted to diagnose cracks and caries in the teeth by using a light source in the near-infrared region. ${ }^{8-14}$ Recently, studies have been introduced to implement images of teeth inside using terahertz waves. ${ }^{15}$ In addition, research on optical coherence tomography devices capable of diagnosing biological tissues such as gums as well as inside teeth has been actively conducted. Mainly, OCT images were conducted to diagnose various diseases such as dental caries, cracks, and resin repair, as well as oral cancer. ${ }^{16}$

In this paper, I would like to review various studies that I studied using optical technology in the dental field. First, it is an oral camera technology that implements images inside the oral cavity using optical fiber lighting technology. Introducing the oral camera technology that is less sensitive to temperature, using optical fiber lighting to address the problem of temperature rise of LEDs, and also acquires fluorescence images. Therefore, an oral camera that can diagnose dental caries was introduced. Second, it is a study of a 3D intraoral scanner produced using a confocal microscope in the 3D structure of a tooth. In order to compensate for the noise problem, a disadvantage of the method of mechanically changing the focus of the lens, the studied oral scanner used a variable focus lens and acquired 3D images using pattern light. In addition, we introduced a study in which several acquired three-dimensional images were stitched to successfully acquire a full-arch image of a tooth. Finally, if the existing 3D scanner was able to acquire only a structural image of the surface of the tooth, it could be diagnosed by acquir- ing a 3D structural image of the tooth as well as a tomographic image inside the tooth using optical tomography technique.

\section{INTRAORAL CAMERA BASED ON THE OPTICAL FIBER LIGHTENING}

A schematic diagram and image of the fluorescence intraoral camera with fiber lightening is shown in Fig. 1. The fluorescence intraoral camera consisted of fiber lightening, camera module with lens and band pass filter. As fiber lightening of the intraoral camera had low temperature, discomfort of the patients due to high temperature of LED lightening could be solved

Temperature of the conventional intraoral camera with LED module and implemented fluorescence intraoral camera with fiber lightening were measured by using IR camera. Fig. 2 is the IR camera images of conventional intraoral camera with the LEDs (a) and implemented fluorescence intraoral camera with fiber lightening (b) after 10 minutes operating. The temperature of the conventional intraoral camera surroundings LED module was about 50-65 degree Celsius. On the contrary, the temperature of fiber lightening was about 25 degree Celsius and was maintained at room temperature without a change in temperature.

Camera module (USB 2M-HU-201, Huentek, Korea) used for experiments had CMOS image sensor, maximum resolution of $1632 \times 1232$ and 2 Mega pixels. Lens

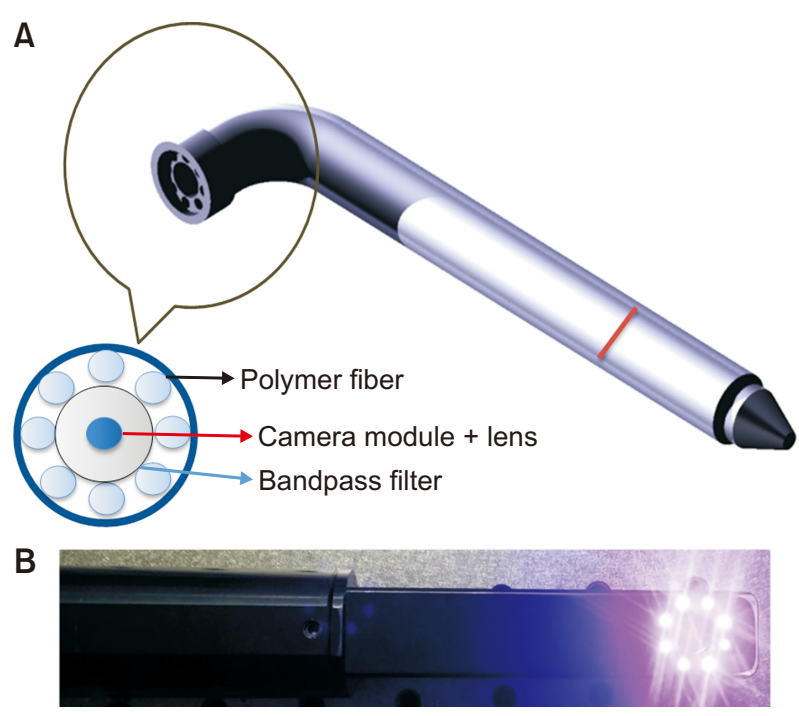

Fig. 1. Schematic of fluorescence intraoral camera with fiber lightening (A) and picture of implemented fluorescence intraoral camera (B). 

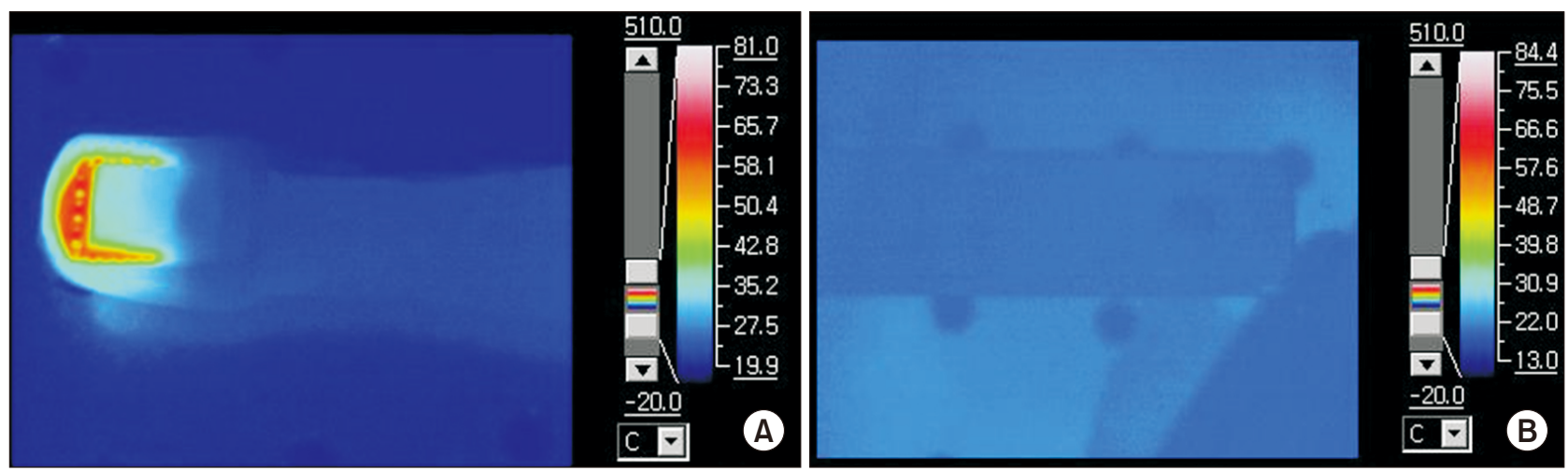

Fig. 2. IR camera images of conventional intraoral camera and fabricated fluorescence intraoral camera with fiber lightening.

with focal length of $10 \mathrm{~mm}$ was placed in the camera module for close-up of the human teeth. Fluorescence intraoral camera with fiber lightening has 8 channel fiber lightening emitting $405 \mathrm{~nm}$ wavelength surrounding camera module with lens and filter and connects to the operatory computer via a USB port and is operatory by software. Fig. 3A show the fluorescence image of the human tooth which got through the proposed fluorescence intraoral camera with fiber lightening. Areas of the tooth that are seen as bright green depicted regions of healthy tooth. While dark areas depicting loss of fluorescence is areas of concentrations of caries or demineralization status of tooth/ The image-processing software quantifies the black and green components of the fluorescence. After green channel image was extracted from image taken by fluorescence intraoral camera shown in Fig. 3B, edge was extracted from green channel image shown in Fig. 3C. Edge clearly was made by using line mask shown in Fig. 3D and generated hole due to extracting edge was filled shown in Fig 3E. Finally, as due to extracting, generated noises and border were removed by imaging process software shown in Fig. 3F, we could identify potential caries in the earliest stages by using fluorescence intraoral camera with fiber lightening. Fig. 4 is fluorescence diagnosis real time image and white light image with on screen orange color after image processing.

\section{D INTRAORAL SCANNING SYSTEM USING TUNABLE LENS AND PATTERN MASK}

Fig. 5 depicts the proposed 3D intraoral scanner. The system comprises four modules: a light source to project structured light onto the sample, a camera module to acquire images of the structured-illuminated sample, a tunable-focus module to vary the focal plane along the focus axis, and a mirror tip module capable of $90^{\circ}$ angle
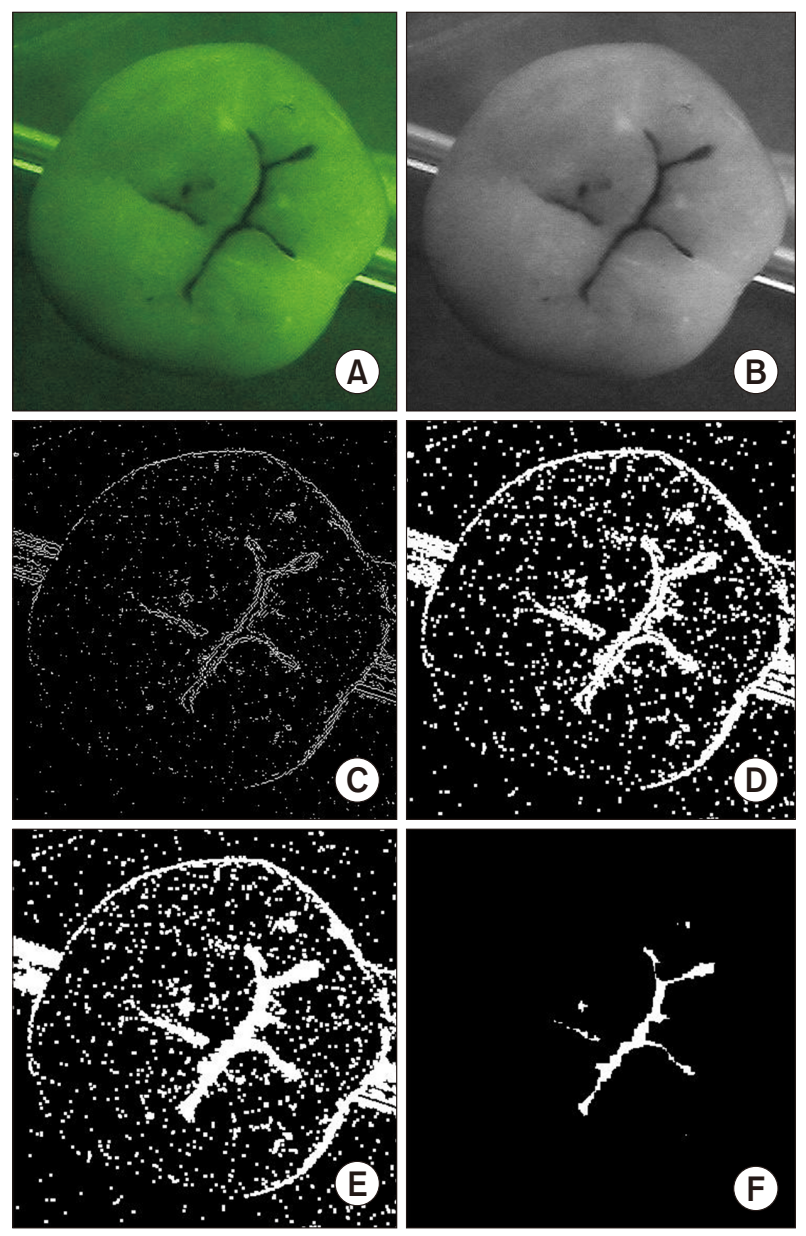

Fig. 3. Dental GUI algorithm. Fluorescence image of human teeth (A); extracted green channel image from color image (B); edge extraction from green channel image (C); edge sharpening by using line mask (D); generated due to edge extraction hole filling $(\mathrm{E})$; border and noise removing $(\mathrm{F})$. 
acquisition. The light source was a white light-emitting diode (LED) (MCEP-CW8-079-3, Moritex, Saitama, Japan). The lens collimates the light from the LED, and a linear polarizer was used to select the vertically polarized component of the illumination source. The collimated light was illuminated to a pattern mask, and we used Ronchi ruling (1-inch × 1-inch (20 lp/mm), Ronchi ruling \#58-777, Edmund Optics, USA) of 50- $\mathrm{mm}$ periodicity as the pattern mask in the experiments. The position of the Ronchi ruling is located at the same distance as the distance of the camera from the lens L1. The structured light passes through the polarizing beam splitter, enters the tunablefocus lens (EL-10-30-C, Optotune AG) through the lens $L 1$, passes through the lens $L 2$, passes through the quarter waveplate (Z-25.4-A-.250-N-556, Tower Optical Corporation, USA), and reaches the sample. The quarter waveplate was arranged at an angle of about 8 degrees to reduce reflection, as the quarter waveplate was not coated with anti-reflection. To eliminate internal reflection from the beam splitter and to improve the contrast ratio of the image, a cross-polarization detection technique
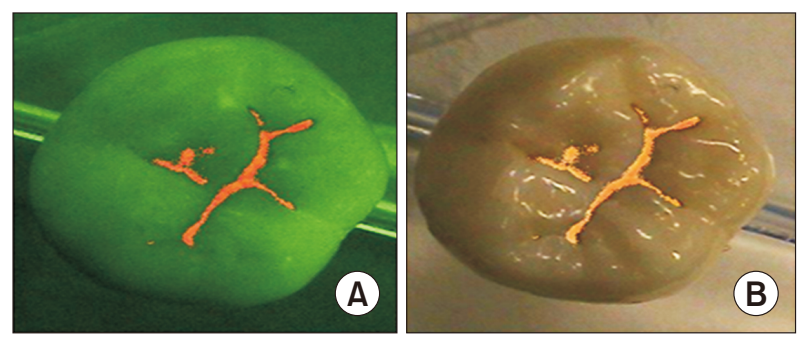

Fig. 4. Fluorescence real time image (A) and white light image (B) of the human tooth after image processing. Fluorescence intraoral camera with fiber lightening could identify suspicious dental caries of human teeth through the different color. was used.

In the experiments, we scanned the dental cast to confirm the feasibility of the proposed intraoral scanner. Fig. 6 shows a picture of the dental cast (Fig. 6A), and 3D point clouds (Figs. 6B-E) acquired from different points of view. The field of view (FOV) of the proposed system was $10.7 \mathrm{~mm} \times 10.7 \mathrm{~mm}$, where the pixel size of the camera is $7.6 \mu \mathrm{m} \times 7.6 \mu \mathrm{m}$ and the size of images acquired by the camera is $1408 \times 1408$ pixels. The depth FOV was $10 \mathrm{~mm}$, and 50 images were acquired by varying the focal planes at a uniform interval. Therefore, the axial resolution of the proposed system was approximately $200 \mu \mathrm{m}$. The precision of the system was evaluated by measuring the gauge block of $2 \mathrm{~mm}$ (18006, Mitutoyo, Japan) 10 times and comparing the three-dimensional coordinate values of the measurement data. The measured precision was 2 $\mathrm{mm} \pm 10.39 \mu \mathrm{m}$.

The image acquisition step immediately acquires the next images without waiting for the completion of the 3D point cloud generation step. As the $500 \mathrm{fps}$ high-speed camera was used, and 50 images were acquired to reconstruct a point cloud in the experiments, the proposed system extracted 10 point clouds per second owing to multithreaded processing.

To confirm the functionality of the intraoral scanner with the fixed pattern mask, we scanned the full arch of the dental cast by performing 3D stitching, which merges the partially overlapped point clouds, using an iterative closest point (ICP) algorithm. ${ }^{17}$ The ICP algorithm was implemented based on the point cloud library. ${ }^{18}$ Fig. 7 shows the 3D point cloud for the full arch of the dental cast. Based on the reconstructed full arch of the dental cast, we can infer that the proposed system has the potential for use in dentistry.

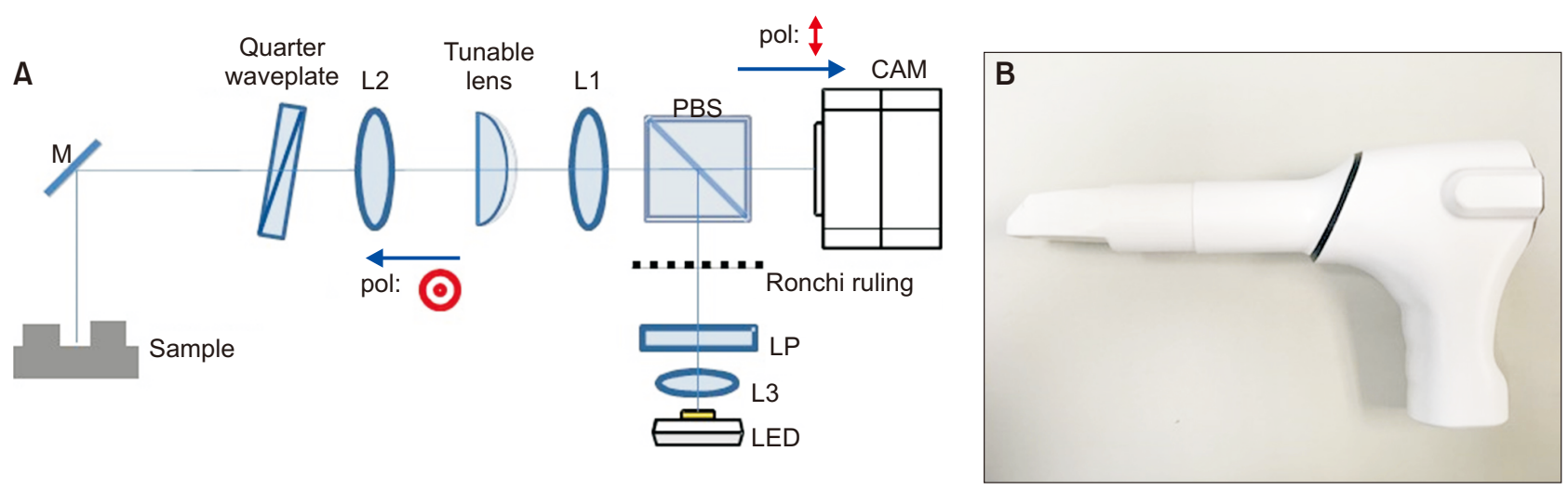

Fig. 5. (A) Schematic of the experimental setup. The $\odot$ and red double-headed arrow represent the polarization of illuminated and scattered light, respectively. (M, mirror; TL, tunable lens; L, lens; PBS, polarizing beam splitter; CAM, camera; LP, linear polarizer). (B) Picture of the prototype 3D intraoral scanner. 


\section{OPTICAL COHERENCE TOMOGRAPHY INTRAORAL SCANNER FOR STRUCTURAL AND FUNCTIONAL DIAGNOSES}

Fig. 8 depicts the SS-OCT system, which was constructed based on a Mach-Zehnder interferometer. The system is equipped with a MEMS-based scan probe to be used inside the mouth. The system comprises five modules: a wavelength swept source, an optical fiber interferometer, a MEMS mirror, a controller, and a digitizer, used for acquiring and displaying OCT volume images. Since the fast scanning speed and enhanced light penetration to the deeper layer were required to utilize the OCT system as an intraoral scan probe, a wavelength swept laser source (Thorlabs, SL1310V1-20048, USA) with a central wavelength of $1310 \mathrm{~nm}$, a scanning rate of $200 \mathrm{kHz}$, and a full-width-at-half-maximum (FWHM) bandwidth of 100

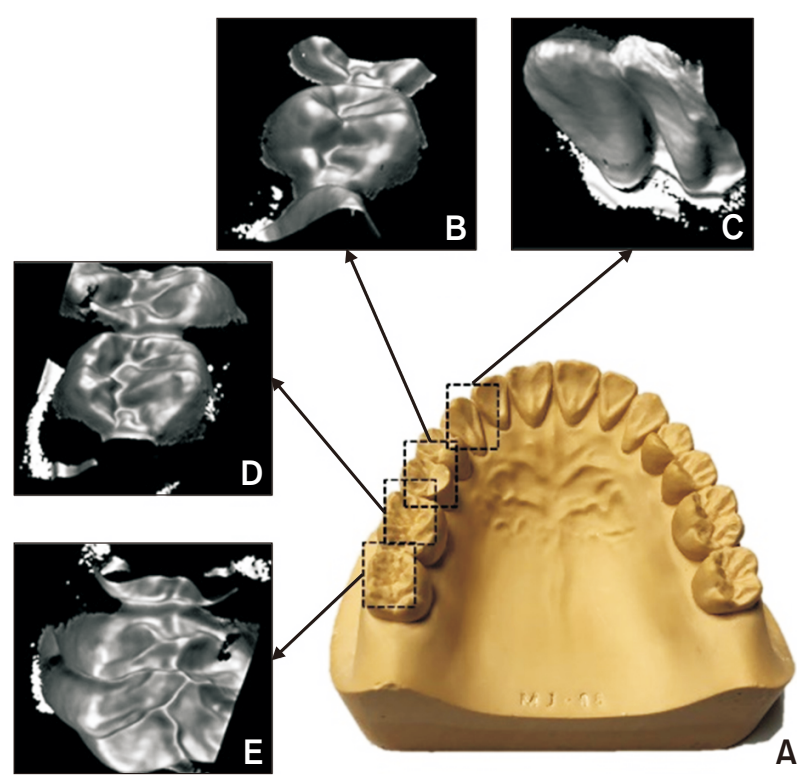

Fig. 6. Dental cast (A) and 3D point clouds (B-E) scanned from the different points of view. $\mathrm{nm}$, is used as the light source. The laser beam from the swept source is guided to the optical fiber Mach-Zehnder interferometer as shown in Fig. 8, which is divided into a sample arm and a reference arm through a 90:10 fiber coupler (Thorlabs, TW1300R2A2, USA).

Fig. 9 shows examples of $2 \mathrm{D}$ cross-sectional images (Fig. 9A) and a 3D volume image (Fig. 9B), which are acquired by the OCT system that was equipped with the MEMS-based scan probe. The scan range was approximately $10 \times 10 \mathrm{~mm}^{2}$. The number of A-scan data samples was 1000 , and the $2 \mathrm{D}$ cross-sectional image was constructed using $500 \mathrm{~A}$-scans, while the 3D volume image comprised 500 cross-sectional images. In other words, the size of the volume acquired by the OCT system was 1000 (A-scan) $\times 500$ (B-scan) $\times 500$ (C-scan). The axial resolution of the system was measured to be $10 \mu \mathrm{m}$ in air. Using a swept laser with a swept source at $200 \mathrm{kHz}$, the frame rate of $2 \mathrm{D}$ cross-sectional images was approximately $780 \mathrm{fps}$, and one 3D volume image was acquired within $0.35 \mathrm{~s}$.

The goal of the intraoral scanner is to digitally impress the specific areas of the teeth and gums that the dentists will treat. To scan the areas-whose dimensions are beyond the FOV of the MEMS-based intraoral scan probepartially overlapped volume. The stitching of 3D volume images is implemented based on an ICP algorithm. ${ }^{17}$ Since the ICP algorithm takes two-point clouds as an input, the point cloud must be extracted from each volume image. Herein, the set of the coordinates of the voxels that have higher intensities than a predefined threshold is considered as the point cloud. Fig. 10 shows point clouds extracted from the 3D volume images of Fig. 10B.

To confirm the applicability of an intraoral scanner based on the OCT system equipped with a MEMS-based intraoral scan probe, the OCT volume images of the extracted human teeth were sequentially acquired, as shown in Fig. 11A-D. The overlapping percentage of the sequentially acquired OCT volumes was more than $70 \%$
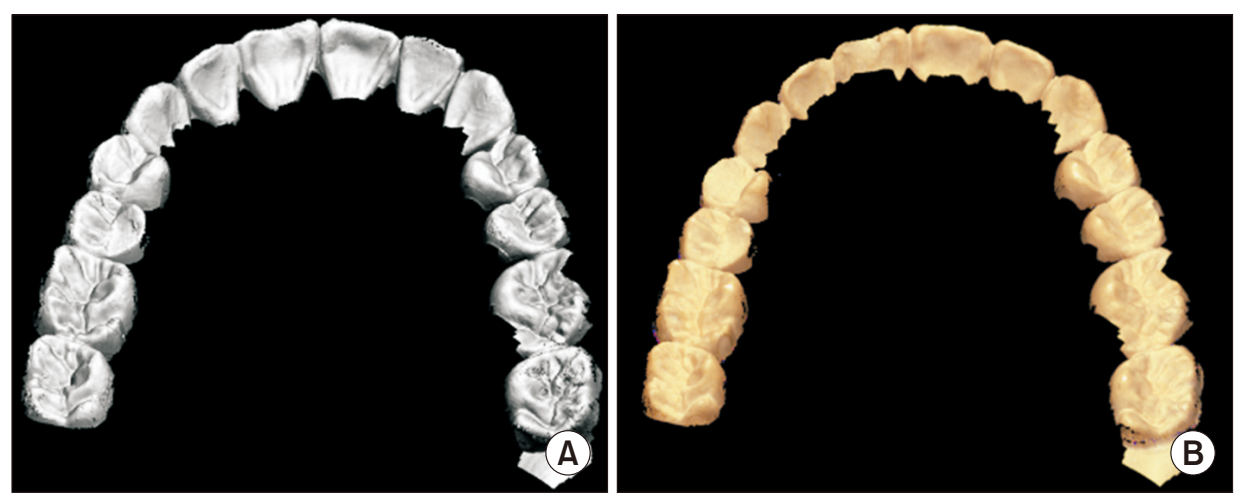

Fig. 7. 3D point cloud of full arch of the dental cast (Fig. 6A) scanned by the proposed system; (A) monochrome image and (B) color image. 


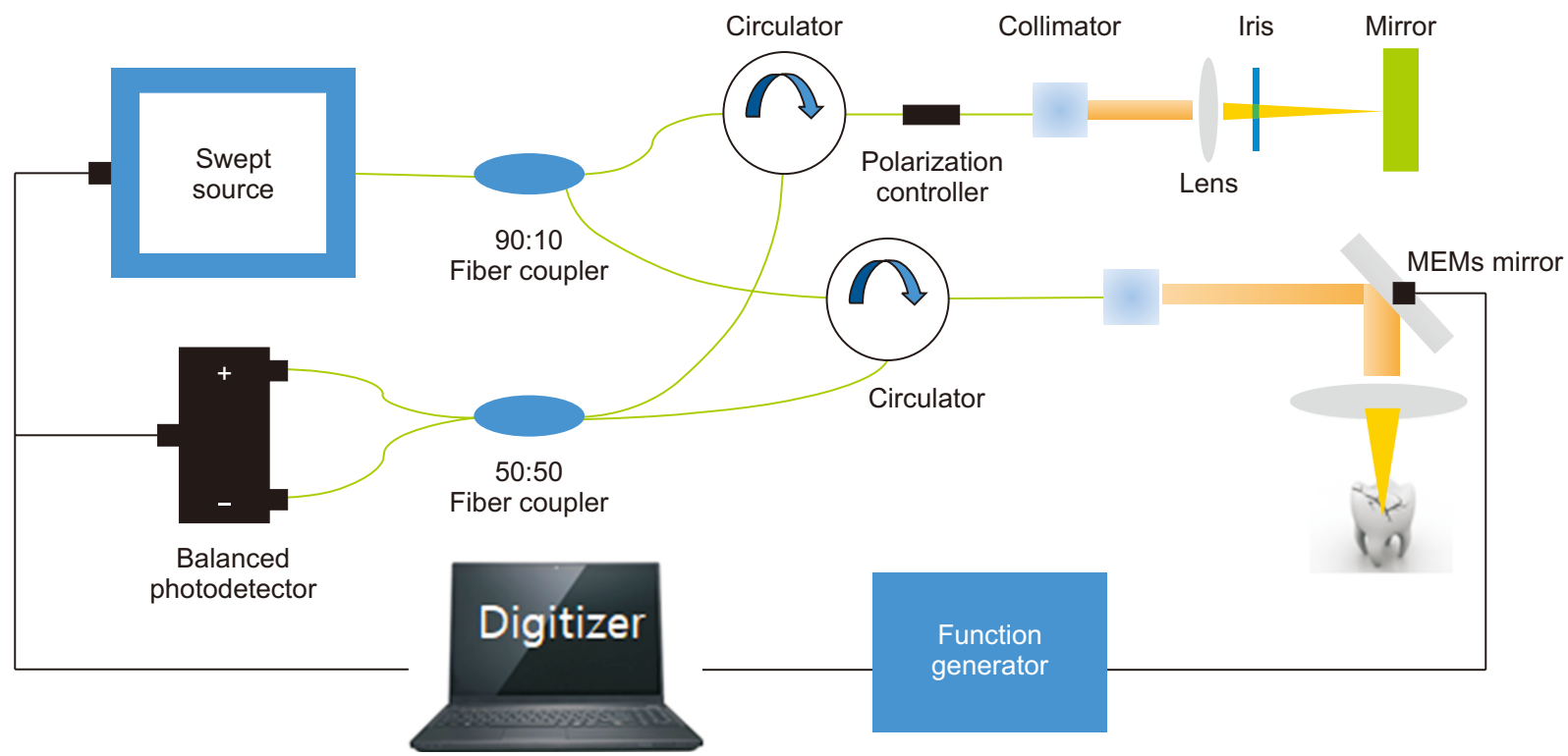

Fig. 8. Schematic of SS-OCT system with MEMS-based scan probe.
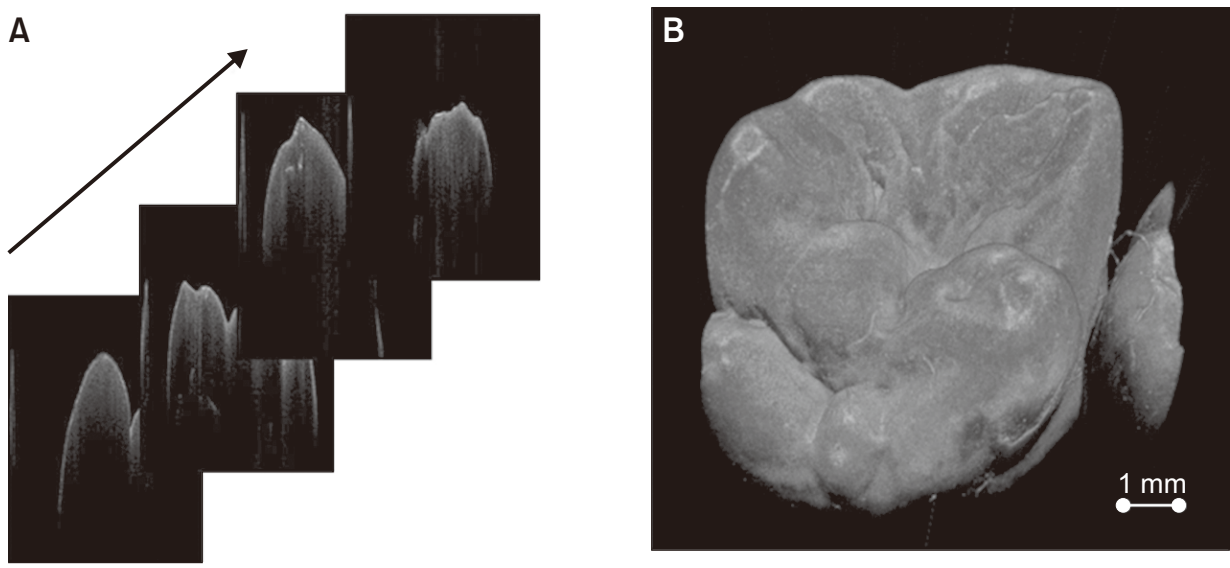

Fig. 9. 2D cross-sectional images (A) and 3D volume image (B) of human tooth acquired by the implemented OCT system.

in the conducted experiments. Fig. 11E-H show point clouds extracted from the OCT volume of Fig. 11A-D. As shown in Fig. 11E-H, voxels located at the surface of the teeth were extracted as the point cloud since the voxel intensities at the surface had relatively higher values than those in other regions of the teeth. Therefore, the set of coordinates of the voxels having higher intensity than a predefined threshold was considered as the point cloud.

The point clouds were used to calculate the rotation matrix and translation vector between two adjacent volumes using the ICP algorithm. The stitching algorithm was implemented based on the point cloud library. The average processing time for pairwise stitching was approximately $0.3 \mathrm{~s}$ on a desktop equipped with a $2.6 \mathrm{GHz}$ Intel Core i7 processor. Fig. 111-K shows 3D stitching results for Fig. 11A-D, based on the rotation matrix and translation vector. Fig. 11 I shows the outcome volume when the volume of Fig. 11B is transformed into the volume of Fig. 11A. Correspondingly, Fig. 11J shows the volume outcome when the volume of Fig. $11 \mathrm{C}$ is transformed into the volume of Fig. 11B, while Fig. 11K shows the outcome when the volume of Fig. 11D is transformed into the volume of Fig. $11 \mathrm{C}$

Fig. 12B shows an example of stitching of OCT volumes that were successively acquired from extracted human teeth, and Fig. $12 \mathrm{C}$ is a three-dimensional image of the enlarged tooth of Fig. 12B. The image of the stitched $3 \mathrm{D}$ volume contains $2 \mathrm{D}$ cross-sectional information of all parts of the teeth. Fig. 12A shows 2D images reconstructed in the XY-plane, while Fig. 12D shows 2D images 

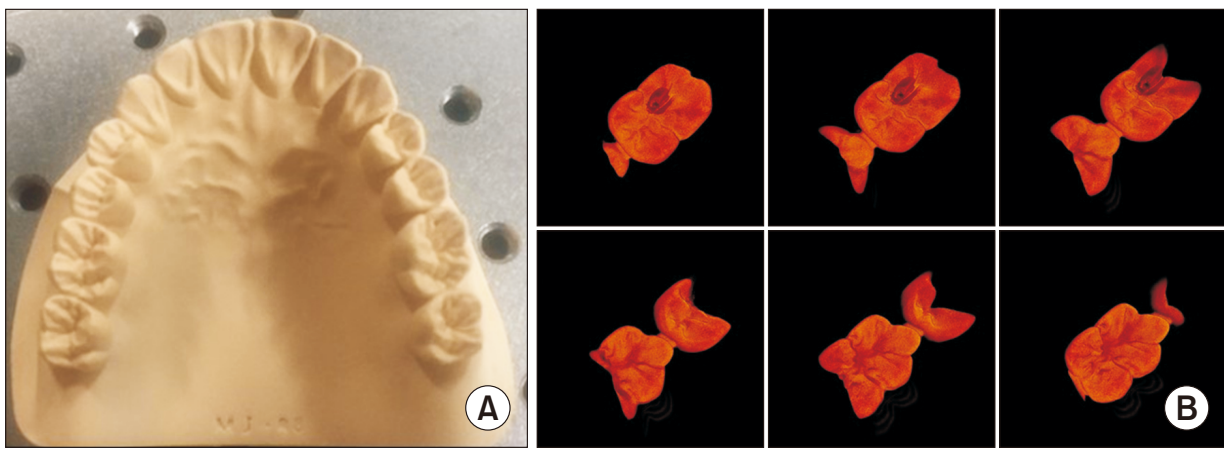

Fig. 10. Examples of $3 \mathrm{D}$ volume images acquired by the OCT scanner: (A) oral teeth model, and (B) sequentially acquired $3 \mathrm{D}$ volume images.
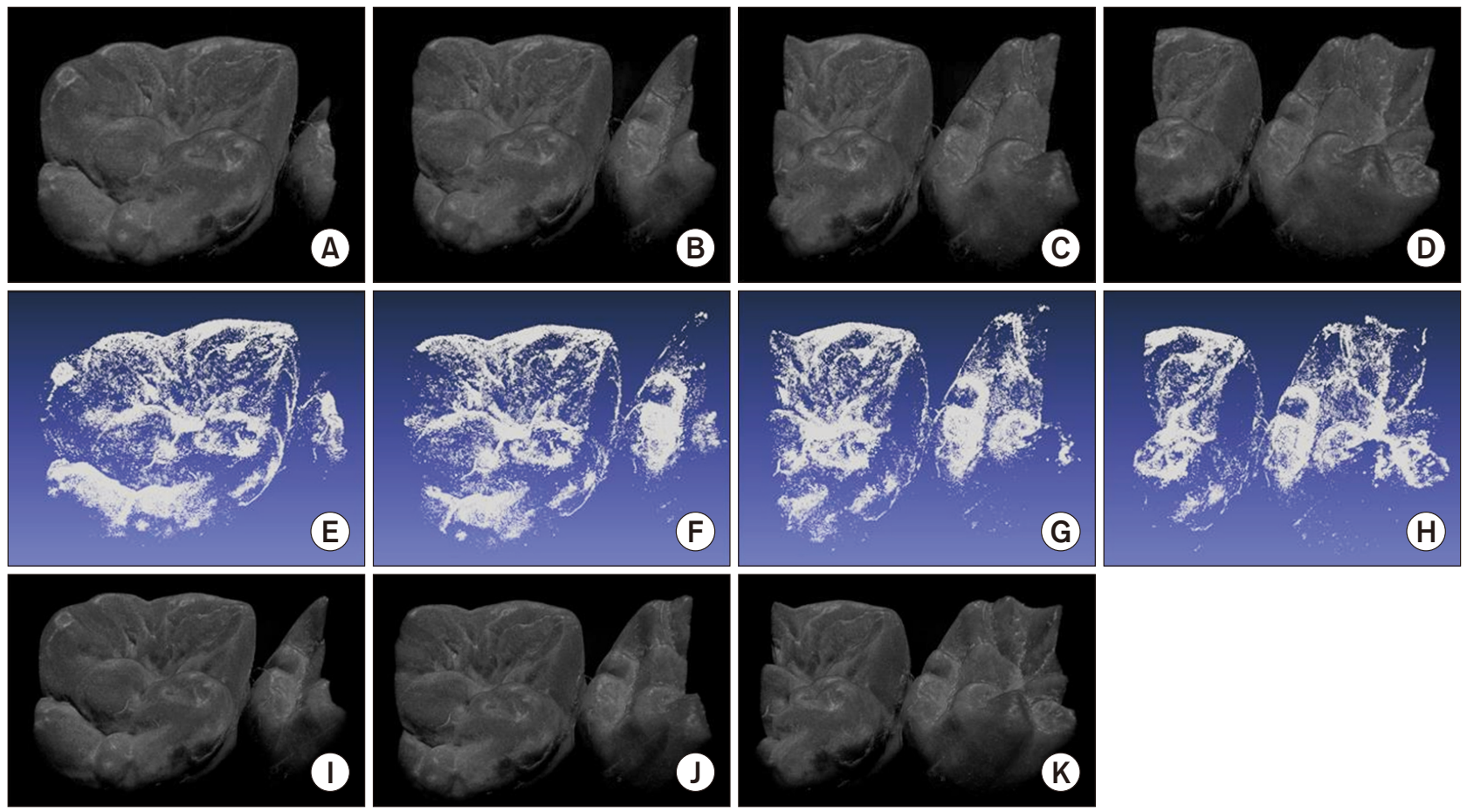

(H)

Fig. 11. Stitching results for partially overlapped OCT volumes: (A-D) sequentially acquired OCT volumes, (E-H) corresponding point clouds, and (I-K) results of 3D stitching of two adjacent volumes.

reconstructed in the XZ-plane. As it can be seen from the $2 \mathrm{D}$ cross-sectional images, the presence of crack, tooth decay, and teeth resin treatment, can be confirmed. In this way, the 3D intraoral scanner based on OCT can acquire 3D structural and diagnostic images of the teeth. It is expected to be extensively used in future diagnoses and teeth treatments.

\section{CONCLUSIONS}

We have introduced methods of diagnosis in dentistry using optical methods. Using a fiber optic light source, the problem of temperature in the oral cavity of the LED was solved, as well as a device for diagnosing cavities us- ing the spontaneous fluorescence characteristics of the tooth. In addition, by using a confocal type polarization optical system and a focus variable lens, the noise problem generated by the conventional oral camera was solved, and a 3D image was obtained using a fixed pattern. In addition, by using the cross polarization method, it has succeeded in acquiring images of highly reflective parts such as metal. Lastly, most of the three-dimensional images of the surface, and the disadvantages of the oral scanner having the function of acquiring only the threedimensional structure, through the optical coherence tomography technique, acquired the internal images of the teeth to enable diagnosis, and at the same time threedimensional Since images can be acquired, the possibility 

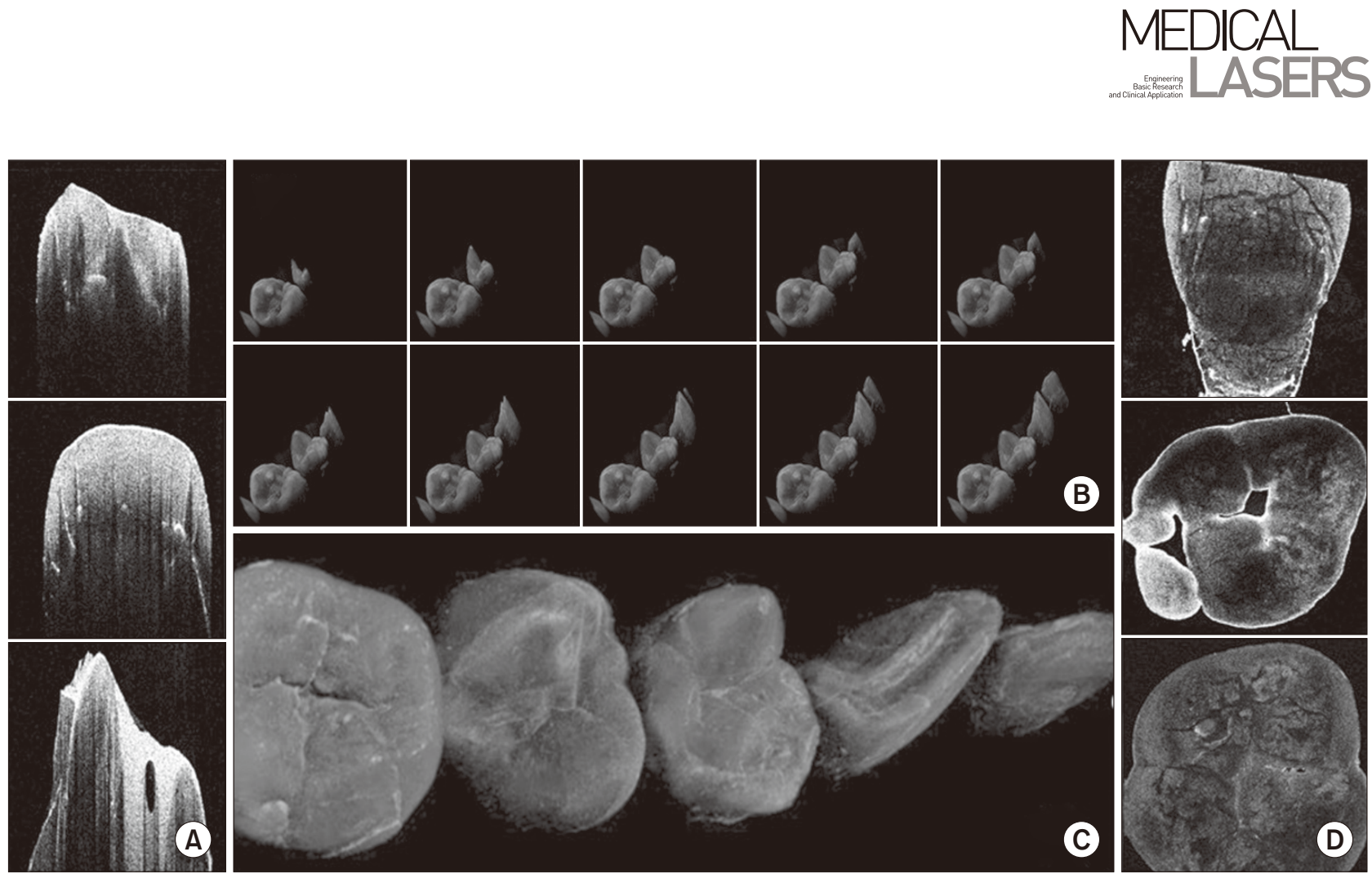

Fig. 12. Results of stitching extracted human teeth acquired by implementing an intraoral scanner based on OCT: (A) 2D cross-sectional images in the XY-plane, (B) stitching result for 10 sequentially acquired volumes, and (C) 2D cross-sectional images in the XZ-plane.

of use as a next-generation oral scanner is increased. As suggested, the optical imaging method is harmless to the human body, has high resolution, and is capable of checking images in real time, so it is expected to be used as a diagnostic device in dentistry.

\section{REFERENCES}

1. de Paula AB, Campos JA, Diniz MB, Hebling J, Rodrigues JA. In situ and in vitro comparison of laser fluorescence with visual inspection in detecting occlusal caries lesions. Lasers Med Sci 2011;26:1-5.

2. Bader JD, Shugars DA. A systematic review of the performance of a laser fluorescence device for detecting caries. J Am Dent Assoc 2004;135:1413-26.

3. Stookey GK, Isaacs RL, Ferreira Zandona AG, Ando M, Gonzalez $C$, et al. Clinical validation of the use of fluorescence for the early detection of dental caries. In: John D. B. Featherstone, Peter Rechmann D.D.S., Daniel Fried, editor(s). Proceedings Volume 3593, Lasers in Dentistry V. BIOS '99 International Biomedical Optics Symposium; 1999 Jan 23-29; San Jose, CA, USA.

4. Amaechi BT. Emerging technologies for diagnosis of dental caries: the road so far. J Appl Phys 2009;105:102047.

5. Pretty IA. Caries detection and diagnosis: novel technologies. J Dent 2006:34:727-39.

6. Qin $Y L$, Luan XL, Bi LJ, Lü Z, Sheng YQ, Somesfalean G, et al. Real-time detection of dental calculus by blue-LEDinduced fluorescence spectroscopy. J Photochem Photobiol B 2007;87:88-94

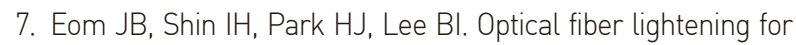
fluorescence signal detection with thermal stability in dentistry. J Biomed Sci Eng 2015;8:201-6.

8. Logozzo S, Zanetti EM, Franceschini G, Kilpelä A, Mäkynen A. Recent advances in dental optics- Part I: 3D intraoral scanners for restorative dentistry. Opt Lasers Eng 2014;54:203-21.

9. Birnbaum NS, Aaronson HB, Stevens C, Cohen B. 3D digital scanners: a high-tech approach to more accurate dental impressions. Insid Dent 2009;5:70-4.

10. Beuer F, Schweiger J, Edelhoff D. Digital dentistry: an overview of recent developments for CAD/CAM generated restorations. Br Dent J 2008;204:505-11.

11. Eom JB, Ahn J, Park A. 3D intraoral scanning system using fixed pattern mask and tunable-focus lens. Meas Sci Technol 2020;31:015401.

12. Ahn JS, Park A, Kim JW, Lee BH, Eom JB. Development of three-dimensional dental scanning apparatus using structured illumination. Sensors (Basel) 2017;17:1634.

13. Hsieh YS, Ho YC, Lee SY, Chuang CC, Tsai J, Lin KF, et al. Dental optical coherence tomography. Sensors (Basel) 2013;13:8928-49.

14. Schneider H, Park KJ, Häfer M, Rüger C, Schmalz G, Krause F, et al. Dental applications of optical coherence tomography (OCT) in cariology. Appl Sci 2017;7:472. 
15. D’Arco A, Di Fabrizio M, Dolci V, Petrarca M, Lupi S. THz pulsed imaging in biomedical applications. Condens Matter 2020;5:25.

16. Eom JB, Ahn JS, Eom J, Park A. Wide field of view optical coherence tomography for structural and functional diagnoses in dentistry. J Biomed Opt 2018;23:1-8

17. BesL PJ, McKay ND. A method for registration of 3-D shapes. IEEE Trans Pattern Anal Mach Intell 1992;14:239-56.

18. Holz D, Ichim AE, Tombari F, Rusu RB, Behnke S. Registration with the point cloud library: a modular framework for aligning in 3-D. IEEE Robot Autom Mag 2015;22:110-24.

How to cite this article: Eom JB, Park A. Applications of optical imaging system in dentistry. Med Lasers 2020;9:25-33. https://doi.org/10.25289/ML.2020.9.1.25 